\title{
Radiation-Induced Growth Retardation and Microstructural and Metabolite Abnormalities in the Hippocampus
}

\author{
Shaefali P. Rodgers, ${ }^{1}$ Janice A. Zawaski, ${ }^{2}$ Iman Sahnoune, ${ }^{1}$ \\ J. Leigh Leasure, ${ }^{1,3}$ and $M$. Waleed Gaber ${ }^{2}$ \\ ${ }^{1}$ Department of Psychology, University of Houston, Houston, TX 77204, USA \\ ${ }^{2}$ Hematology-Oncology Section, Department of Pediatrics, Baylor College of Medicine, Houston, TX 77030, USA \\ ${ }^{3}$ Department of Biology \& Biochemistry, University of Houston, Houston, TX 77204, USA \\ Correspondence should be addressed to M. Waleed Gaber; gaber@bcm.edu
}

Received 27 November 2015; Revised 11 February 2016; Accepted 5 April 2016

Academic Editor: Michela Buglione

Copyright (C) 2016 Shaefali P. Rodgers et al. This is an open access article distributed under the Creative Commons Attribution License, which permits unrestricted use, distribution, and reproduction in any medium, provided the original work is properly cited.

Cranial radiotherapy (CRT) increases survival in pediatric brain-tumor patients but can cause deleterious effects. This study evaluates the acute and long-term impact of CRT delivered during childhood/adolescence on the brain and body using a rodent model. Rats received CRT, either $4 \mathrm{~Gy}$ fractions $\times 5 \mathrm{~d}$ (fractionated) or a cumulative dose of $20 \mathrm{~Gy}$ (single dose) at $28 \mathrm{~d}$ of age. Animals were euthanized $1 \mathrm{~d}, 5 \mathrm{~d}$, or $3.5 \mathrm{mo}$ after CRT. The $3.5 \mathrm{mo}$ group was imaged prior to euthanasia. At $3.5 \mathrm{mo}$, we observed significant growth retardation in irradiated animals, versus controls, and the effects of single dose on brain and body weights were more severe than fractionated. Acutely single dose significantly reduced body weight but increased brain weight, whereas fractionation significantly reduced brain but not body weights, versus controls. CRT suppressed cell proliferation in the hippocampal subgranular zone acutely. Fractional anisotropy (FA) in the fimbria was significantly lower in the single dose versus controls. Hippocampal metabolite levels were significantly altered in the single dose animals, reflecting a heightened state of inflammation that was absent in the fractionated. Our findings indicate that despite the differences in severity between the doses they both demonstrated an effect on cell proliferation and growth retardation, important factors in pediatric CRT.

\section{Introduction}

Radiation, as a monotherapy or as a component of a polytherapeutic approach, is used to treat malignant cancers due to its high degree of efficacy. Unfortunately, radiation does not distinguish among the types of cells it destroys, and so healthy tissues in and around the cancerous ones are also compromised [1]. Thus, survival carries with it a risk of deleterious side effects as a result of radiation-induced normal brain tissue injury. This presents a unique challenge with regard to the pediatric population, wherein cranial radiotherapy (CRT) is part of treatment for acute lymphoblastic leukemia (ALL) and solid tumors of the brain, which comprise a significant proportion of malignant cancers [2]. The nonspecific radiation-induced perturbations to the developing brain can have adverse consequences, both acute and long-term, for the brain and body [3-9]. Clinical imaging studies provide evidence at the functional network level that CRT has detectable effects on cerebral integrity [10-12], connectivity [13-15], and volume [11, 16-21]. Numerous studies have found changes in metabolites and/or diffusion tensor parameters in the brain after irradiation in rodents. Using magnetic resonance spectroscopy changes in $\mathrm{N}$-acetylaspartate (NAA), glutamate, choline, lactate, taurine, and myo-inositol have been measured in different brain regions after radiation exposure [22-24]. In addition, diffusion tensor imaging (DTI) parameters have been measured in a variety of brain regions, such as the fimbria, external capsule, and corpus callosum, after brain irradiation [23-26]. In this study, we employ these imaging measurements to investigate the effect of CRT on adolescent rats. CRT also disrupts endocrine function by suppressing growth hormone expression [27-29] resulting in growth retardation $[9,30]$ and significant alterations in body composition and weight are often observed in survivors of 
radiation-treated childhood cancers [7, 31, 32]. Although there is a great deal of individual variability within survivors in terms of diagnosis, sex, age at treatment, and type, dose, and regimen of treatment, childhood CRT is recognized as being the biggest risk factor for negative neurocognitive and psychosocial outcomes in adulthood, leading to a poorer quality of life overall $[33,34]$. As such, it is imperative to characterize the scope and precise mechanisms of CRT-induced damage in order to determine how best to minimize the costbenefit ratio of CRT and establish effective interventions to mitigate late-onset neurocognitive deficits that are generally irreversible.

It is widely accepted that the adverse response of the normal tissue in the brain to single dose irradiation occurs early, while its response to fractionated irradiation is late. Differences in the expression levels of inflammatory molecules, between single and fractionated doses, have been reported [35]. However, most of these studies have focused on the early phase of radiation damage and little has been done to elucidate the long-term difference between these two regimens. Animal models of pediatric radiotherapy provide the opportunity to examine radiation effects at the cellular, structural, and circuit levels in the developing brain. One of the most devastating effects of radiation is on cell genesis. Animal studies of single dose radiation effects on neurogenesis show a dramatic decrease [36-38], but the effects of fractionated irradiation of the adolescent brain on hippocampal cell genesis have been less studied. In this work, we investigated the effects of single dose and fractionated CRT in adolescent rats on body weight, brain weight, Ki67 (a marker of cell proliferation), and fractional anisotropy (a measure of functional integrity of fiber tracts) in the fimbria fornix as well as metabolite changes in the hippocampus. The hippocampus and its primary efferent projection (the fimbria fornix) are critical for learning and memory $[39,40]$. Indeed, it is thought that the cognitive impairments associated with cancer treatment are due in large part to effects on this structure [4143]. In this paper, we will present data on proliferation, microstructural, and metabolite changes measured in these two structures.

\section{Methods}

2.1. Subjects. Fifty-five male Wistar rats were purchased from Harlan Sprague Dawley Inc. (Indianapolis, IN). Animal care was in accordance with guidelines set by the National Institutes of Health Guide for the Care and Use of Laboratory Animals (2011) and all procedures were approved by the Institutional Animal Care and Use Committee of Baylor College of Medicine (Houston, TX). Rats were 24 days old upon arrival and were housed in pairs in a temperature-controlled vivarium under a 12-hour dark/light cycle (lights off 7:00 am7:00 pm) with unrestricted access to food and water. Body weights were recorded daily during the course of radiation and then weekly and prior to euthanasia for brain harvest. All irradiated rats developed malocclusions involving their upper incisors. These abnormalities took approximately 6 weeks after radiation to manifest. At this point, their teeth were trimmed under isoflurane anesthesia once every week until termination of the experiment. This was also when their weekly weights were recorded. Sham animals were concurrently anesthetized and weighed to control for exposure to isoflurane. The overgrowth of the incisors prevented irradiated animals from being able to consume the standard, hard, food pellets. Therefore, all rats, including shams, were given fresh, water-softened food pellets in their home cages, every day, throughout the course of the experiment. This was initiated immediately after the first session of CRT to ensure that the drop in body weight during the course of radiation was not due to a CRT-induced inability to chew the standard pellets or fatigue/illness that would prevent only the irradiated animals from accessing the pellets on top of their home cages.

2.2. Irradiation Procedure. At 28 days of age, animals were anesthetized using isoflurane and randomly assigned to either the radiotherapy (CRT) or sham group. Animals in the CRT group were individually irradiated at a dose rate of $128 \mathrm{cGy} / \mathrm{min}$ using a RS 2000 biological X-ray irradiator $150 \mathrm{kVp}, 25 \mathrm{~mA}$ (Rad Source Technologies, Inc., Suwanee, GA). Each rat was placed prone and lead shielding was used to ensure that only the region of the head beginning behind the eyes and extending to approximately $5 \mathrm{~mm}$ behind the ears received radiation, so that the whole brain (cerebrum and cerebellum), but not the eyes, would be within the field of radiation. Approximately half of the CRT animals received a single dose of $20 \mathrm{~Gy}(n=19)$ and the other half received the same dose divided into $4 \mathrm{~Gy}$ fractions across 5 consecutive days $(n=18)$. Animals in the sham group $(n=18)$ were anesthetized for the same length of time as those in the CRT group but did not receive radiation. Animals from each group were sacrificed $1 \mathrm{~d}$ ( $n=6$ per group), $5 \mathrm{~d}$ ( $n=6$ per group), and $3.5 \mathrm{mo}$ ( $n=6 / 7$ per group) following start of CRT (Figure 1).

2.3. Magnetic Resonance Imaging (MRI). Animals euthanized at the $3.5 \mathrm{mo}$ after CRT interval were first imaged approximately between 3 and 3.5 mo after CRT (Figure 1). A 9.4T Biospec MRI scanner (Bruker, MA) with a $20 \mathrm{~cm}$ bore and a quadrature rat brain array (Bruker, MA) were used. All animals underwent spectroscopy followed by DTI. Animals were anesthetized with isoflurane and placed prone on the imaging bed. A respiratory pillow was placed under the abdomen of the animals and a rectal probe was used to monitor respiration and temperature, respectively. The quadrature rat brain array was centered and fixed over the rat brain. Initially, a Tripilot scan was performed to optimize animal placement within the magnet. A T2-rapid acquisition with relaxation enhancement (T2-RARE) scan with the following parameters, $\mathrm{TE}=20 \mathrm{~ms}$, TR $=2600 \mathrm{~ms}$, \# of averages $=1$, $3 \mathrm{~cm}$ field of view, 12 slices, $1 \mathrm{~mm}$ slice thickness, $1.1 \mathrm{~mm}$ interslice distance, and $256 \times 256$ matrix size, was then performed and used to localize the placement of the spectroscopy voxel. Voxel placement was in the hippocampus $(2 \mathrm{~mm}$ height, $4 \mathrm{~mm}$ width, $3 \mathrm{~mm}$ anterior to posterior). Prior to MR spectroscopy a FASTMAP sequence was performed to correct for local field inhomogeneity by adjusting first- and secondorder shim coil currents. All MR spectra acquired using a stimulated echo (STEAM) sequence with the following parameters, $\mathrm{TR}=2 \mathrm{~s}, \mathrm{TE}=2.22 \mathrm{~ms}$, \# of averages $=512$, and 


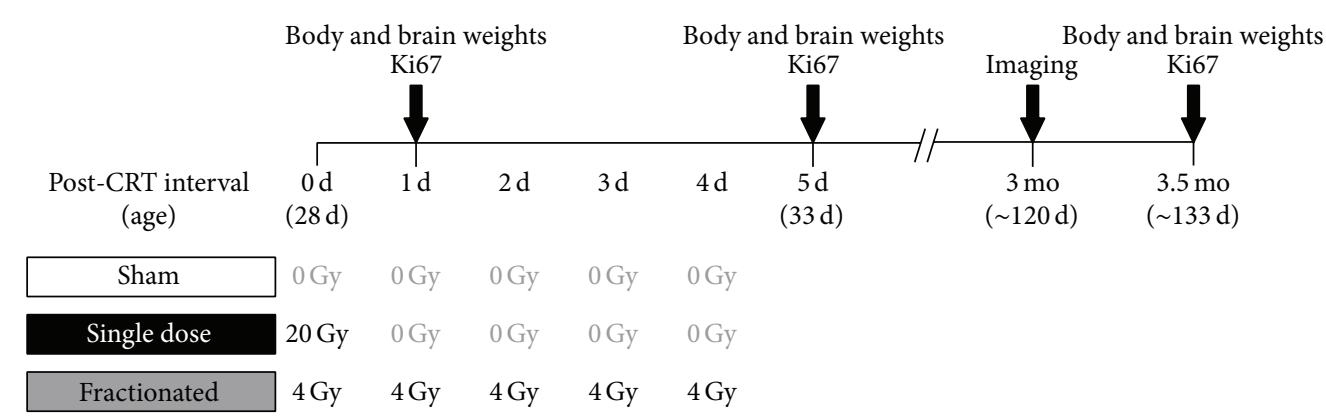

FIGURE 1: Experiment design and timeline. Note that animals were euthanized at $1 \mathrm{~d}, 5 \mathrm{~d}$, and 3.5 mo, making this a between-group design.

voxel size $=24 \mathrm{~mm}^{3}$, were executed. Water suppression was achieved using variable pulse power and optimized relaxation delay (VAPOR). For quantification purposes, two scans were obtained, with and without water suppression. MR spectra were then analyzed using LCModel (LCModel, Canada). Metabolites were excluded when they did not pass the criterion of $n \geq 4$ /group and Cramér-Rao lower bounds $<20 \%$.

Following spectroscopy, DTI was performed $(n=6-7 /$ group) using a spin echo planar imaging (EPI) sequence with $\mathrm{a} \mathrm{TR}=500 \mathrm{~ms}, \mathrm{TE}=33.2 \mathrm{~ms}, 2$ repetitions, slice thickness = $12.8 \mathrm{~mm}, b$ value $=800 \mathrm{~s} / \mathrm{mm}^{2}$, \# of directions 30 , and matrix size $128 \times 256 \times 16$ giving a spatial resolution of $150 \mu \mathrm{m} \times$ $150 \mu \mathrm{m} \times 800 \mu \mathrm{m}^{3}$. Total imaging time, for spectroscopy and DTI, was approximately 1 hour per animal. Fractional anisotropy (FA) maps were processed through Medinria and analyzed using Inveon Research Workstation (Siemens, Washington, DC). Briefly, in slices in which the fimbria fornix was visible, a region of interest (ROI) was drawn around the fimbria fornix (left and right sides) and a FA threshold value of $\geq 0.6$ and $\geq 0.75$ applied and mean FA calculated. Mean FA values obtained were averaged between left and fight fimbria and between slices for each animal. The change in fimbria volume caused by radiation was calculated as follows. First, total fimbria volume (TFV) was calculated using a $0.5 \mathrm{FA}$ threshold, to isolate the entire fimbria (Figure 4(a)), in each imaging slice in which the fimbria was visible. Second, a FA threshold value of $\geq 0.6$ and $\geq 0.75$ was applied to the TFV and a resulting thresholded volume $\left(\mathrm{TV}_{0.6}, \mathrm{TV}_{0.75}\right)$ was calculated (Figure 4(b)). Third, the TVX was calculated and averaged between left and fight fimbria and between slices for each animal to arrive at $\sum \mathrm{TV}_{X}$. Finally, a ratio of $\sum \mathrm{TV}_{X} / \mathrm{TFV}$ was calculated (Figure $4(\mathrm{c})$ ).

2.4. Brain Tissue Harvest and Processing. Animals were given an overdose of ketamine/xylazine (i.p.) and transcardially perfused with $4 \%$ paraformaldehyde at $1 \mathrm{~d}, 5 \mathrm{~d}$, or $3.5 \mathrm{mo}$ after the start of CRT (Figure 1). Brains were removed, postfixed for $24 \mathrm{~h}$, and stored in 30\% sucrose until sectioned. Brains (forebrain and cerebellum only) were weighed and then cut into $50 \mu \mathrm{m}$ serial, coronal sections on a freezing-stage sliding microtome (Leica Microsystems, SM2000R, Nussloch, Germany) and stored in cryoprotectant in 96-well microtiter plates at $-20^{\circ} \mathrm{C}$. Every 6 th section throughout the rostrocaudal extent of the hippocampus was processed by standard immunohistochemical procedure as reported previously [45] to label proliferating cells. Briefly, sections were rinsed in $0.1 \mathrm{M}$ tris-buffered saline (TBS), quenched for $30 \mathrm{~min}$ at room temperature in $0.6 \%$ hydrogen peroxide, and rinsed in TBS again. Next, sections were pretreated in $10 \mathrm{mM}$ sodium citrate buffer ( $\mathrm{pH} 8.5$ ) for $30 \mathrm{~min}$ in a $80^{\circ} \mathrm{C}$ water bath, allowed to cool down to room temperature, and rinsed in TBS before incubation in 3\% normal donkey serum (Sigma-Aldrich, St. Louis, MO) for $1 \mathrm{~h}$. This was followed by incubation at $4^{\circ} \mathrm{C}$ for $72 \mathrm{~h}$ in primary antibody (rabbit, polyclonal antiKi67, 1: 1800; Vector Laboratories, Burlingame, CA). Sections were then rinsed in TBS, blocked in 3\% normal donkey serum for $15 \mathrm{~min}$, and incubated overnight at room temperature in secondary antibody (biotinylated donkey, antirabbit, 1:250; Jackson ImmunoResearch Laboratories, West Grove, PA). Next, sections were rinsed in TBS, treated for $90 \mathrm{~min}$ in avidin-biotin complex (ABC, Vector Laboratories), and rinsed again. Sections were reacted and visualized with diaminobenzidine, rinsed before being mounted onto gelatin-coated slides, and allowed to dry overnight. Sections were then counterstained with methyl green, cleared in xylene, and cover-slipped using Permount mounting medium (Fisher Scientific, Pittsburgh, PA).

2.5. Histological Analysis. Ki67+ cells were quantified in the subgranular zone (SGZ) of the dentate gyrus (DG) using a Nikon Eclipse 80i upright microscope in conjunction with StereoInvestigator (MicroBrightField, Williston, VT). Approximately, 10-11 sections per animal were analyzed for Ki67+ cells at 40x magnification throughout the septotemporal axis of the hippocampus by an experimenter blind to the conditions. Since Ki67 staining was sparse, cells were counted nonstereologically, and the number of cells was summed across sections for each animal.

2.6. Statistical Analyses. All data were analyzed with Prism 6 (GraphPad Software, Inc., La Jolla, CA). Ki67+ cell counts in the SGZ ( $1 \mathrm{~d}, 5 \mathrm{~d}$, and $3.5 \mathrm{mo}$ after CRT), body weights (recorded just before euthanasia at each interval), and normalized brain weights ( $24 \mathrm{~h}$ and $3.5 \mathrm{mo}$ after the end of CRT) were analyzed by two-way ANOVAs with CRT and postCRT interval as factors. One-sample $t$-tests were used to compare differences between mean brain weight per group (at each interval) and shams (i.e., a value of 100). Mean FA values, fimbria volume-ratios per applied threshold, and 


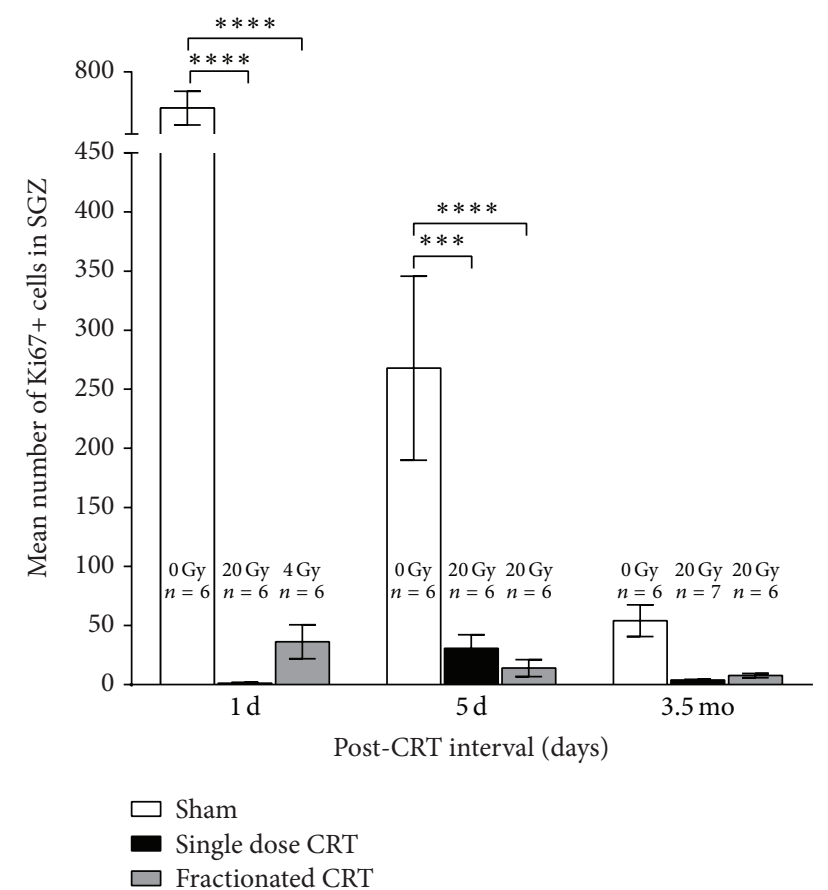

FIGURE 2: Mean number $( \pm$ SEM) of Ki67+ cells in the SGZ $1 \mathrm{~d}$, $5 \mathrm{~d}$, and $3.5 \mathrm{mo}$ after CRT $\left[{ }^{* * *} p<0.001,{ }^{* * * *} p<0.0001\right]$. Note that all animals are age-matched at each post-CRT interval and that 24 hours after the start of radiation the single dose CRT and fractionated CRT groups differ only in terms of the cumulative dose of radiation received. The number of Ki67+ cells dropped significantly with age only in the shams.

hippocampal metabolite levels were analyzed by one-way ANOVAs. Data on body weight, body length, and body weight/length collected from a small, separate cohort of animals (see Figure 6) were analyzed by one-way repeated measures ANOVAs. Bonferroni post hoc comparisons were conducted, where appropriate. Differences between group means were considered significant if $p<0.05$. Graphs represent mean values or individual data points \pm SEM.

\section{Results}

3.1. Cell Proliferation. There was a significant main effect of CRT $[F(2,46)=59.72, p<0.0001]$, post-CRT interval $[F(2,46)=20.13, p<0.0001]$, and interaction $[F(4,46)=$ 17.81, $p<0.0001]$ on the mean number of Ki67+ cells in the SGZ of the hippocampus (Figure 2). Post hoc analyses revealed that single dose CRT as well as fractionated CRT significantly reduced the number of Ki67+ cells relative to shams at the $1 \mathrm{~d}$ and $5 \mathrm{~d}$ post-CRT intervals. However, due to the substantial drop in Ki67+ cells in shams due to age alone [ $1 \mathrm{~d}$ versus $5 \mathrm{~d}$ : $p<0.0001,5 \mathrm{~d}$ versus $3.5 \mathrm{mo}: p=0.001$ ], this difference did not reach statistical significance in adulthood.

3.2. Spectroscopy. In total, 13 metabolites were successfully measured ( $N \geq 4$ /group and $\leq 20 \%$ Cramér-Rao lower bound) in all experimental groups. However, only two metabolites, myo-inositol and $\mathrm{N}$-acetylaspartate $+\mathrm{N}-$ acetylaspartylglutamate (NAA + NAAG), had measureable

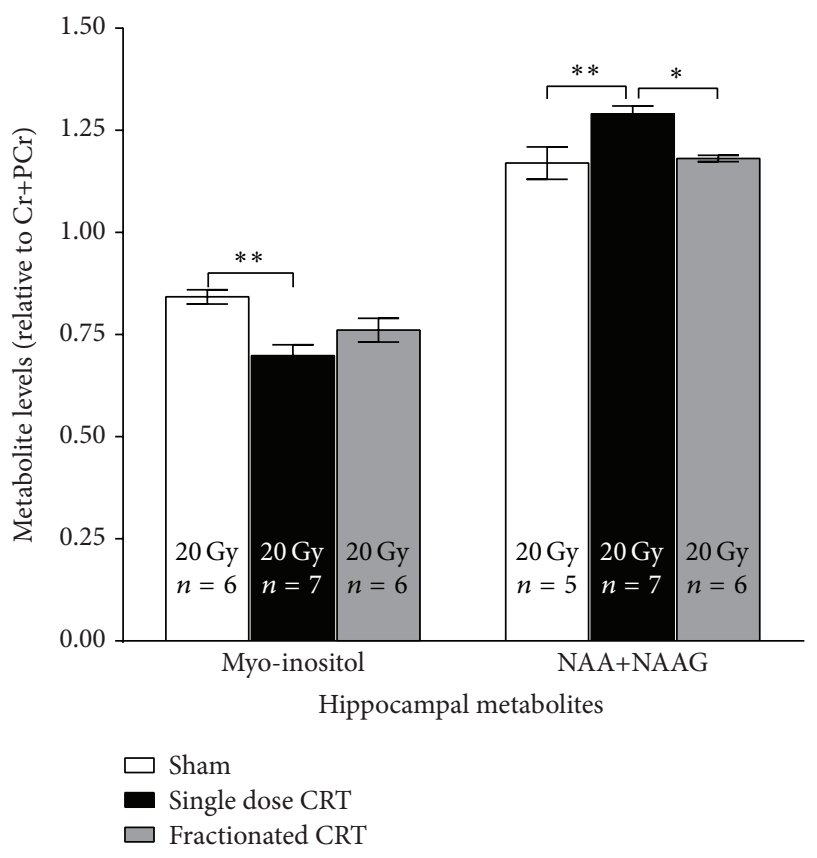

FIGURE 3: Metabolite changes measured in the hippocampal region at 3 mo after CRT. There was a significant decrease in myo-inositol levels in the single dose group compared to sham, while an increase in NAA + NAAG was measured in the single dose group compared to shams and fractioned CRT $\left[{ }^{*} p<0.05,{ }^{* *} p<0.01\right]$.

significant change. There was a significant effect of CRT on myo-inositol $[F(2,16)=8.3, p<0.01]$ and NAA + NAAG levels $[F(2,15)=8.75, p=0.003]$ at $3 \mathrm{mo}$ after CRT (Figure 3). Post hoc analyses showed that myo-inositol was significantly lower in single dose CRT animals compared to shams $[p<0.01]$. NAA + NAAG levels were significantly higher in single dose CRT animals relative to shams $[p<$ $0.01]$ and fractionated CRT animals $[p<0.05]$.

3.3. DTI. At 3 mo after CRT, no changes in mean FA were found in the fractionated CRT animals. However, single dose CRT animals demonstrated a significant reduction compared to shams $[p<0.05]$ in mean FA values within voxels that were equal to or greater than the set threshold of 0.6 FA (Figure 4(b)). Further, post hoc analyses revealed that, relative to shams, only single dose CRT animals had a significant reduction in the ratio of the fimbria volume above FA thresholds of $0.6[p<0.01]$ and $0.75[p<0.05]$ (Figure 4(c)). Furthermore, the ratio of the volume of the fimbria above FA thresholds of 0.6 and 0.75 in fractionated CRT was also reduced, albeit not significantly.

3.4. Body Weights. There was a significant main effect of CRT $[F(2,46)=56.55, p<0.001]$ and post-CRT interval $[F(2,46)=556.9, p<0.001]$ on body weight as well as a significant interaction between CRT and post-CRT interval $[F(4,46)=39.14, p<0.001]$. Post hoc tests revealed that, at 3.5 mo after CRT, both fractionated and single dose-treated animals had significantly reduced body weights relative to age-matched shams $[p<0.0001]$ (Figure 5). In addition, 

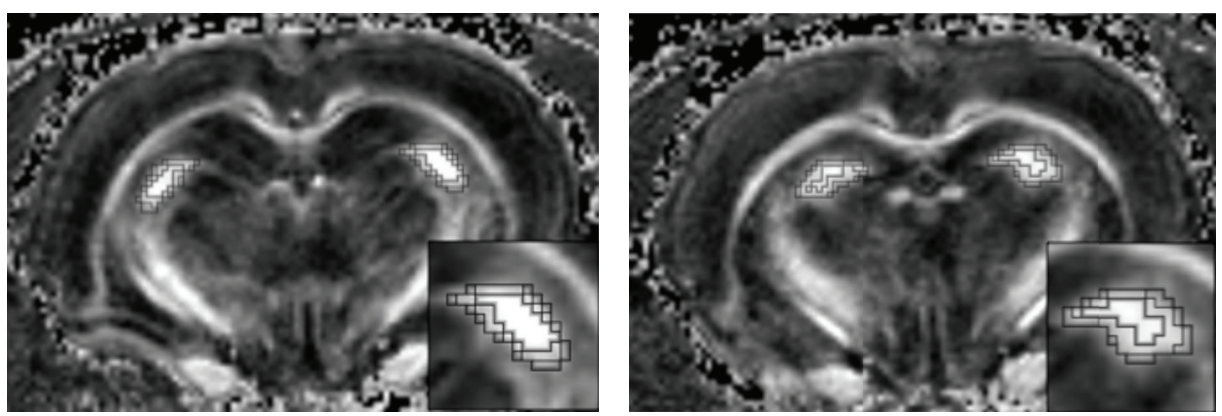

(a)

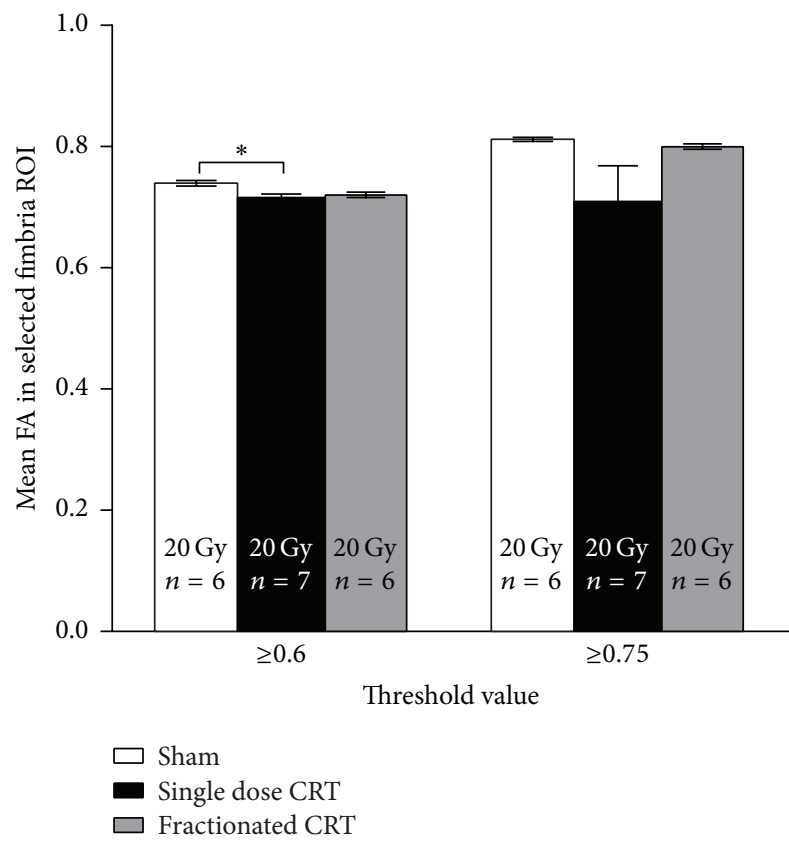

(b)

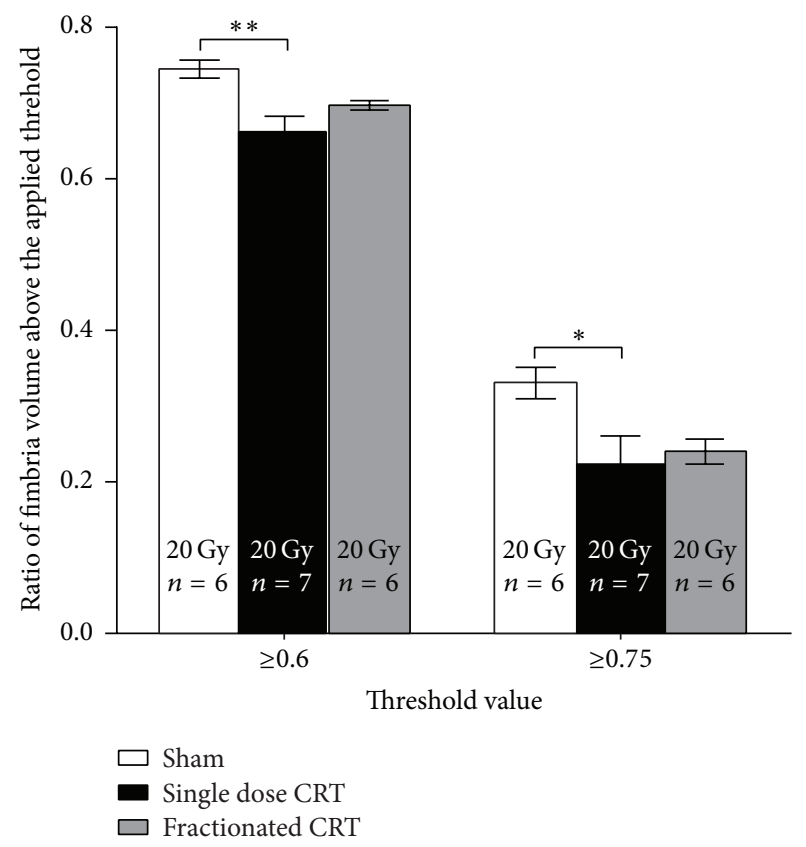

(c)

Figure 4: (a) Representational images of the fimbria in sequential cross sections. Concentric thresholded ROIs, representing thresholds of $0.5,0.6$, and 0.75 successively from large to small. Zoomed in insert has been added to better visualize the ROIs. (b) Mean FA values ( \pm SEM) at 3 mo after CRT in the ROI within the fimbria determined by the threshold value. Note that single dose CRT had a reduction in mean FA in voxels $\geq 0.6$ FA. (c) Ratio of fimbria volumes $( \pm$ SEM) at 3 mo after CRT. There was a reduction in the ratio of the fimbria volume with the applied FA thresholds of $\geq 0.6$ and 0.75 in the single dose CRT animals only [ $\left.{ }^{*} p<0.05,{ }^{* *} p<0.01\right]$.

single dose CRT suppressed body weight even relative to fractionated CRT [ $p<0.001]$. At $5 \mathrm{~d}$ after CRT, that is, $24 \mathrm{~h}$ after the last fractionated dose of radiation, only the single dose CRT group had significantly reduced body weight relative to shams [ $p<0.05]$. We also measured differences in body length and body weight/length ratios (Figure 6).

3.5. Brain Weights. Brain weights of CRT groups were normalized to that of age-matched shams and compared at $24 \mathrm{~h}$ and 3.5 mo following end of treatment (Figure $7(a)$ ). There was a significant effect of interval on brain weight $[F(1,21)=$ 141.1, $p<0.0001]$ and a significant interaction between CRT and interval $[F(1,21)=108.6, p<0.0001]$. Post hoc analyses showed that, the day after end of treatment, single dose CRT significantly increased brain weight relative to shams $(9.2 \%$ increase, $[p<0.01])$ and fractionated CRT $(15.7 \%$ increase, $[p<0.0001])$. However, in the fractionated CRT group, mean brain weight was significantly lower than sham $(6.5 \%$ reduction, $[p=0.0001]) .3 .5 \mathrm{mo}$ after end of treatment, single dose CRT significantly reduced brain weight relative to shams (18.9\% reduction, $[p<0.0001])$ and fractionated CRT $(10.6 \%$ reduction, $[p<0.0001])$. The mean brain weight of the fractionated CRT group was also significantly reduced relative to sham $(8.4 \%$ reduction, $[p=0.0001])$. Mean brain weight of the single dose CRT group was reduced $28.1 \%$ at $3.5 \mathrm{mo}$ relative to $24 \mathrm{~h}$ [ $p<0.0001]$. In contrast, there was no change across time in the fractionated CRT group.

\section{Discussion}

4.1. CRT Suppresses Hippocampal Cell Proliferation. CRTinduced suppression of cell proliferation in the SGZ was evident the day after the start of radiation and persisted into adulthood (Figure 2). There were no significant 


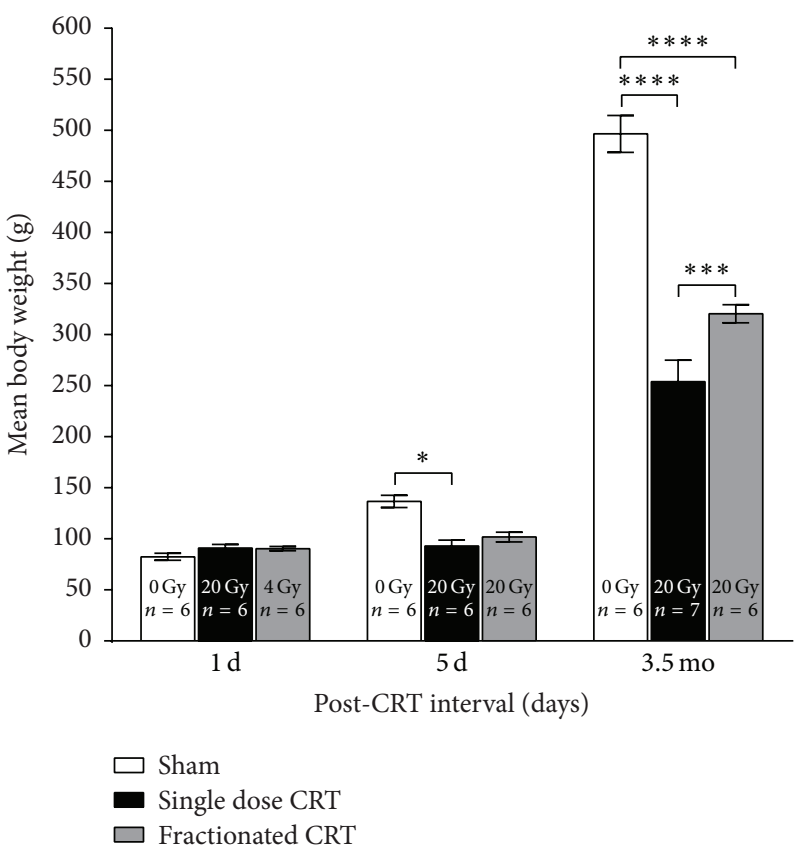

(a)

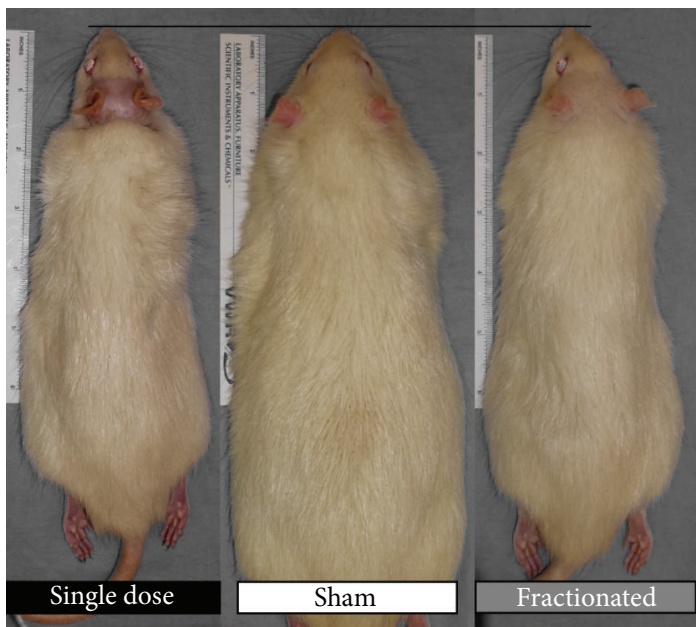

(b)

Figure 5: (a) Mean body weights $\left( \pm\right.$ SEM) $1 \mathrm{~d}, 5 \mathrm{~d}$, and 3.5 mo after CRT $\left[{ }^{*} p<0.05,{ }^{* * *} p<0.001,{ }^{* * * *} p<0.0001\right]$. Note that all animals are age-matched at each post-CRT interval and that 24 hours after the start of radiation the single dose CRT and fractionated CRT groups differ only in terms of the cumulative dose of radiation received. (b) Representative images of a rat from each group 3.5 mo after radiation demonstrate the dramatic retardation of body growth as a consequence of radiation. Note also the permanent effect of single dose radiation on hair growth on the dorsal surface of the region of the head directly in the field of radiation.

differences between single dose and fractionated regimens at any interval, although there were more Ki67+ cells at the $1 \mathrm{~d}$ post-CRT interval in the animals that received a single $4 \mathrm{~Gy}$ fraction relative to those that received a single $20 \mathrm{~Gy}$ dose while this pattern was reversed at $5 \mathrm{~d}$. It may be that there was some recovery in the single dose animals 5 days after their last exposure to radiation or that this is simply an artifact of within-group variance. If assessed independently at each interval, the differences between single dose and fractionated CRT reach statistical significance only at the $1 \mathrm{~d}$ interval which is not surprising as at this point the comparison is between doses of $20 \mathrm{~Gy}$ and $4 \mathrm{~Gy}$. Note that while proliferation in neither irradiated group differed significantly across intervals, the number of Ki67+ cells in shams dropped significantly as a function of age. Our observations concur with those of others reporting a comparable number of Ki67+ cells in the SGZ of naïve rodents of similar age $[38,46]$ and a significant drop in neurogenesis between adolescence and adulthood [47-49]. The decrease in proliferation in shams from $1 \mathrm{~d}$ to $5 \mathrm{~d}$ may in part be a function of error variance and/or due to repeated isoflurane exposure which is reported to suppress hippocampal neurogenesis in juvenile but not in adult rodents $[50,51]$. However, studies on isofluraneinduced neurotoxicity typically use long durations of anesthesia, whereas, in our experiment, animals received, on average, $10-15 \mathrm{~min}(1-3 \%, 0.5-1 \mathrm{~L} / \mathrm{min})$ from days $0-4$. Furthermore, as sham animals were matched to irradiated animals for isoflurane exposure, the differences in proliferation between the irradiated groups and age-matched sham groups cannot be attributed to isoflurane. Our results agree with others [38, 52, 53], as independent ANOVAs for each post-CRT interval reveal a significant main effect of CRT even at $3.5 \mathrm{mo}$ after CRT. However, when the data are analyzed as a whole, the significance is lost. We chose a factorial analysis to control for familywise error rate, but the fact remains that, in the absence of data at earlier time points, we would have detected a statistically significant difference at this time point. In summary, our results indicate that $20 \mathrm{~Gy}$ of radiation, delivered either as a single dose or in 5 daily fractions, suppresses hippocampal cell proliferation acutely relative to controls, and there is no change in proliferation from the acute to early delayed phase after radiation in both CRT groups.

The SGZ, one of the primary neurogenic niches of the brain, contains progenitor cells which proliferate and subsequently migrate to the adjacent granule cell layer whereupon they differentiate and mature. The survival of these neurons is typically determined 11-16 days after mitosis; therefore, any damage sustained during this period could disrupt normal brain development resulting in adverse long-term effects [54]. Our data show that a dose of radiation as low as $4 \mathrm{~Gy}$ is sufficient to suppress cell proliferation to approximately $6 \%$ of control levels, 24 hours after exposure. This is consistent with evidence that the majority of apoptosis among progenitor cells in the SGZ occurs within 24 hours after radiation [54] and other reports of a long-lasting decrease in proliferation or neurogenesis in the rodent hippocampus following cranial radiation in early development [55-58]. Disruption of hippocampal neurogenesis during development, especially if 


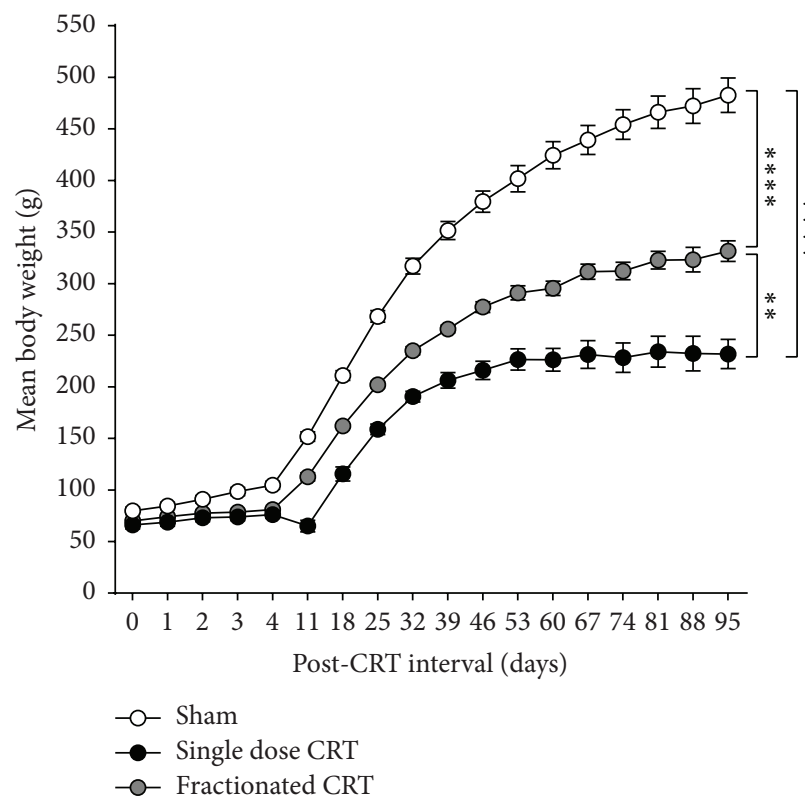

(a)

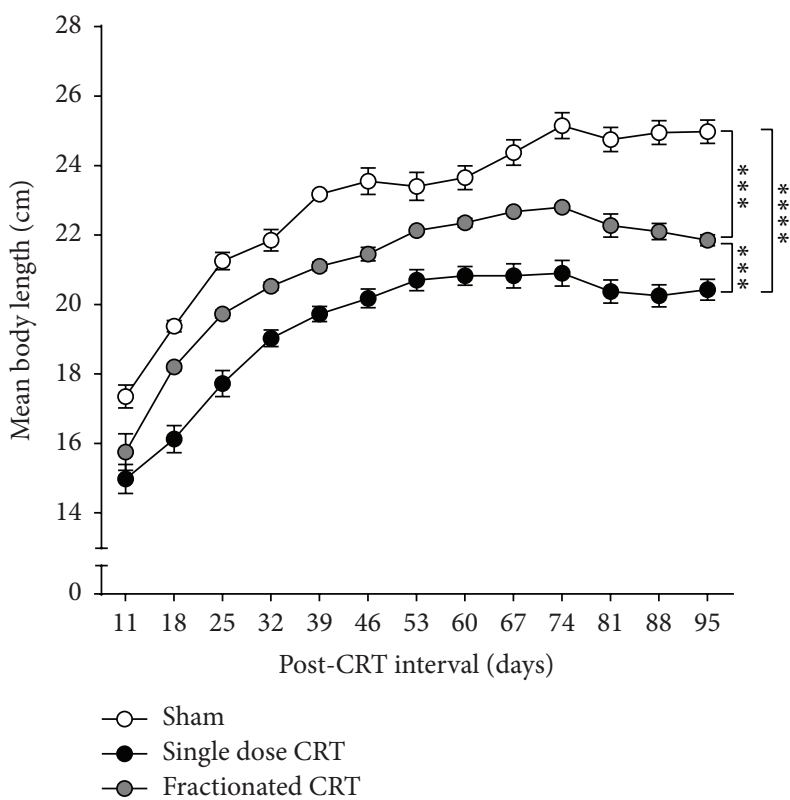

(b)

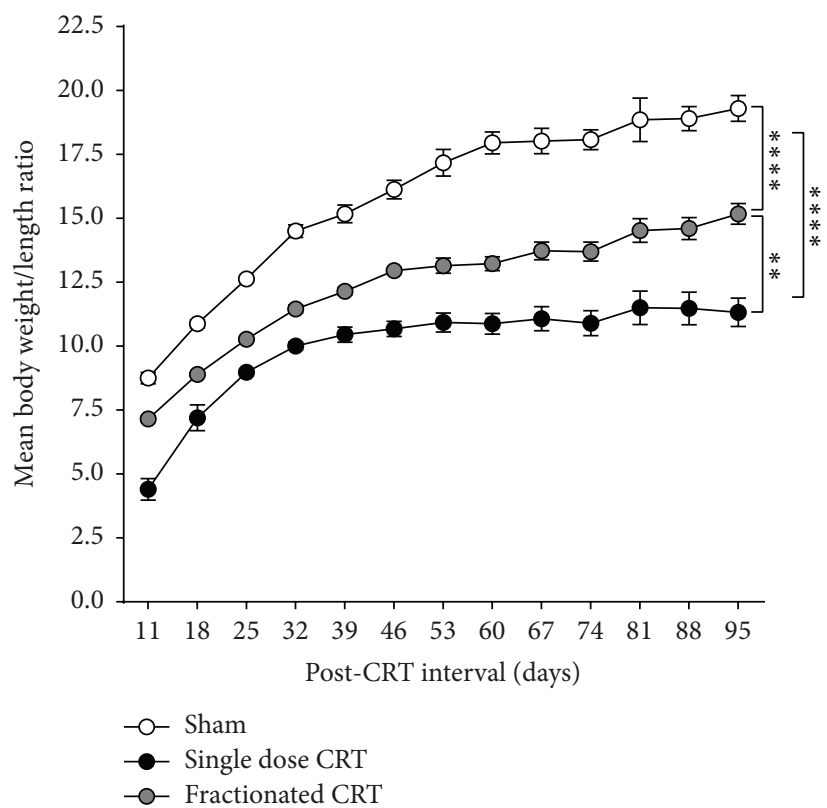

(c)

FIGURE 6: Eight male Wistar rats were irradiated at 28 days of age. They were randomly assigned to single dose CRT $(n=4)$ and fractionated CRT $(n=4)$ and irradiated as described in Methods. Age-matched shams $(n=4)$ were anesthetized but not irradiated. (a) Mean body weights $( \pm$ SEM) before start of radiation (day 0), during the course of radiation (days 1-4), and every week for 12 weeks after CRT. (b) Mean body length $( \pm$ SEM) from nose to tail for 12 weeks after CRT. Animals were placed ventral surface down on a surgical drape, while under anesthesia. Tip of the nose and base of the tail were marked on the drape and the distance between them was measured by the same experimenter throughout. (c) Mean body weight/body length ratio $( \pm \mathrm{SEM})\left[{ }^{* * * *} p<0.0001,{ }^{* * *} p<0.001,{ }^{* *} p<0.01\right]$.

persistent, may well contribute to the decrease in brain size that we have observed $[59,60]$ (Figure $7(b))$ as well as late cognitive sequelae of childhood CRT [43, 61, 62].

\subsection{Microstructural and Metabolite Changes following Single} Dose CRT. To assess radiation-induced damage, we used DTI, a noninvasive MRI technique commonly used to measure the diffusion of water within the brain, which can be altered by organizational or microstructural changes in tissue matter [63]. A decrease in FA value (an increase in unrestricted water movement) in the brain has been correlated with edema, inflammation, and a decrease in neuronal myelination [64]. In our model, a significant decrease in mean FA was measured in the fimbria of animals treated with single 


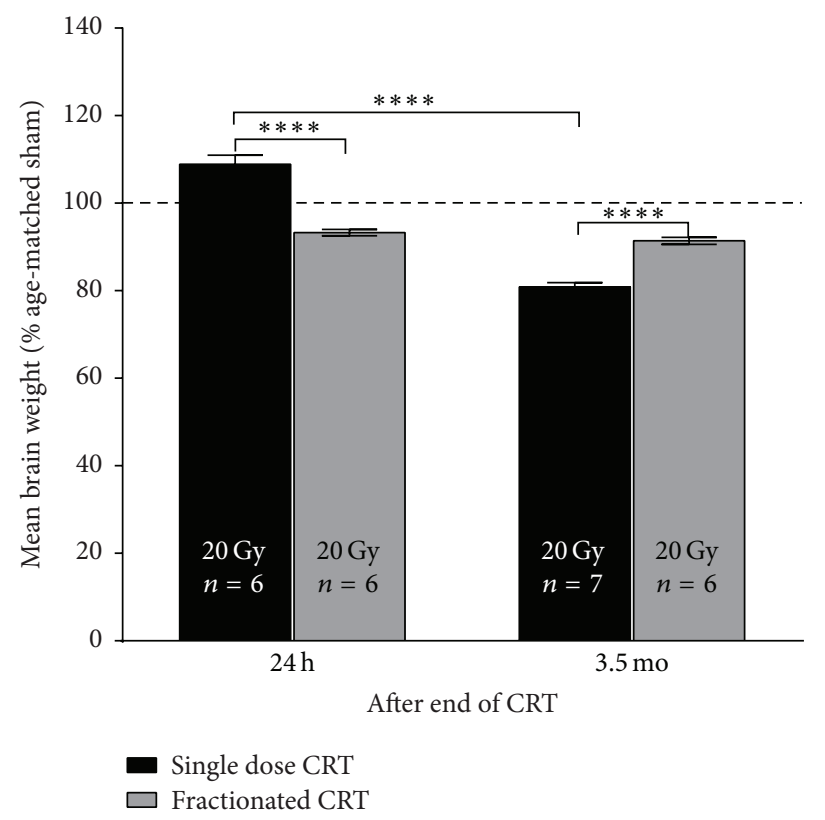

(a)

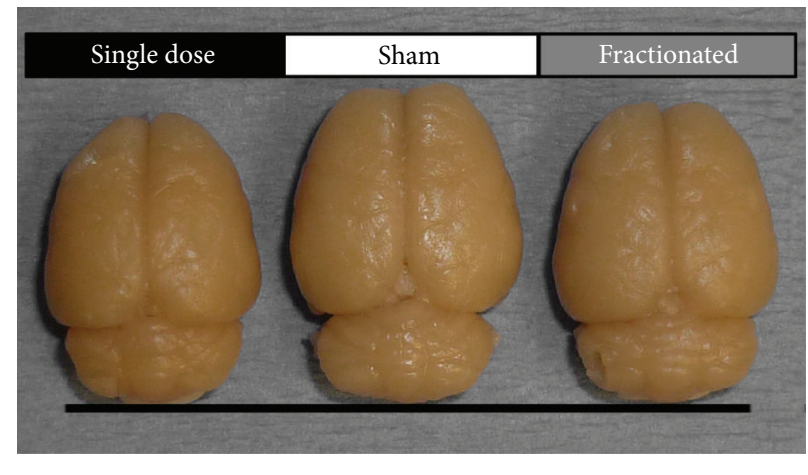

(b)

FIGURE 7: (a) Brain weights expressed as \% of age-matched shams ( \pm SEM) $24 \mathrm{~h}$ and 3.5 mo after end of CRT. The day after end of treatment, fractionated CRT significantly reduced brain weight by $6.5 \%$ compared to shams $[p<0.001$ ]. Single dose CRT, however, significantly increased brain weight by $9.2 \%$ compared to shams $\left[p<0.01\right.$ ] and $15.7 \%$ relative to fractionated CRT [ $\left.{ }^{* * * *} p<0.0001\right] .3 .5$ mo after end of treatment, fractionated CRT significantly reduced brain weight by $8.4 \%$ compared to shams $[p=0.0001$ ] and $10.6 \%$ relative to single dose CRT [ $p<0.0001$ ] which reduced brain weight by $18.9 \%$ [ $p<0.0001]$. Mean brain weight of the single dose CRT group was reduced $28.1 \%$ at 3.5 mo relative to $24 \mathrm{~h}$ [ $p<0.0001$ ]. (b) Representative images of a brain from each group 3.5 mo after radiation highlight the radiation-induced stunting of brain size visible even to the naked eye (figure originally published in [44]).

but not fractionated dose CRT, implying increased radiationinduced damage compared to fractionated CRT. Our findings are consistent with those of others who have reported that FA decreases after single dose radiation exposure in the fimbria $[23,65]$ and other fiber tracts $[24,26,65,66]$ but is not detectable following fractionated treatment [25]. Histological studies also report white matter damage following a single exposure to radiation $[23,67-69]$. We also found that the ratio of fimbria volume was decreased in the single dose-treated animals at both thresholds applied, whereas the fractionated CRT animals showed no reduction at either threshold. Another study using adolescent mice also reported that volumetric differences in the fimbria emerge as early as 1 week after single dose CRT and show limited subsequent recovery [70]. Given that the fimbria fornix comprises a bundle of afferent and efferent myelinated projections to and from the hippocampus, radiation-induced damage to this white matter tract may contribute to late cognitive effects [69].

Using magnetic resonance spectroscopy, we found that, in the single dose, but not in the fractionated, CRT group, myo-inositol decreased significantly, while NAA + NAAG increased significantly relative to shams. These two metabolites are involved in astrocyte metabolism; myo-inositol, which is an osmolyte and is also involved in membrane turnover $[71,72]$, is more abundant in astrocytes than in neurons, and the production and metabolism of NAA + NAAG are through a cycle that involves neurons, astrocytes, and oligodendrocytes $[56,57]$. Our findings agree with those reported by Atwood et al. [73]. Coupling these metabolites findings with our observed reduction in cell proliferation (Figure 2) seems to indicate that in the case of severe radiation-induced injury, as demonstrated by the single dose CRT, the onset of gliosis and demyelination could offer an explanation for these changes. We have previously reported an increase in gliosis following single dose cranial irradiation that persists for months after treatment $[74,75]$. Further, we and others have observed demyelination onset at around three months after irradiation [76, 77]. Further experimentation and maybe the use of NMR spectroscopy of brain tissue samples would be needed to confirm our observation.

4.3. CRT Stunts Growth. Within the first few days after the start of radiation, body weights of irradiated animals were lower than those of age-matched shams (Figure 5(a)). While both regimens suppressed growth, only the difference between single dose and sham reached statistical significance at the $5 \mathrm{~d}$ post-CRT interval, but, by $3.5 \mathrm{mo}$, all three groups differed significantly from one another. We have observed the retarding effect of CRT on body weight in other strains of rats as well (Long-Evans hooded and Fisher 344, data not shown). We also replicated our findings of CRT-induced overall reduction in body weight in a small, separate cohort 
of animals (Figure 6(a)). Single dose CRT animals, again, had the highest reduction in body weight. Note that this cohort was followed over time and when data for $5 \mathrm{~d}$ after CRT (i.e., day 4 on $x$-axis, Figure $6(a)$ ) are analyzed by one-way ANOVA, shams weigh significantly more than both single dose $[p<0.001]$ and fractionated CRT $[p<0.01]$ animals. Additionally, we documented significant effects of CRT, postCRT interval, and CRT x post-CRT interval on body length (Figure 6(b)) and the ratio of body weight to body length (Figure 6(c)). All groups differed significantly from each other in terms of body length and body weight/length ratio and single dose CRT had the most severe impact. Furthermore, while growth eventually plateaued with age for all groups, irradiated animals never recovered to sham levels. However, note that while CRT decreased body weight and length, the rate of weight gain and increase in length was similar across groups. Post-irradiation weight loss in rodents has been reported in mice and rats irradiated with different doses and at different ages [25, 78-81]. In order to mitigate the effects of possible damage to the salivary gland, we provided softened food to the animals inside their home cages on a daily basis, starting immediately after the first session of radiation. However, growth remained stunted even 3.5 mo after treatment.

Our brain weight data at 3.5 mo after radiation show that both single dose and fractionated CRT stunted brain growth (Figure 7(a)). Once again, a single dose of CRT suppressed growth to a significantly larger extent (18.9\% decrease) than fractionated did ( $8.4 \%$ decrease). Brains of single dose CRT animals weighed, on average, $10.6 \%$ less than those of fractionated CRT animals, which is visible to the naked eye (Figure $7($ b)). Other studies have also reported radiation-induced suppression of brain growth in rodents [25, 49, 79, 82, 83]. In contrast, $24 \mathrm{~h}$ after the end of CRT, that is, when both groups had received a total dose of $20 \mathrm{~Gy}$, brains of single dosetreated animals were, on average, heavier compared to shams and fractionated CRT animals, whereas brains of fractionated CRT animals weighed less compared to shams. The initial increase in brain weight among single dose-treated animals may be a result of acute inflammation-induced edema which is absent in the fractionated CRT group, because they receive radiation over 5 days which allows for bouts of recovery between exposures that are 1/5th the dose.

Collectively, our findings show that whole brain radiation has long-lasting consequences for growth, paralleling the clinical literature [84-86], and that despite some differences, primarily related to inflammation, CRT delivered in childhood/adolescence causes growth stunting of the brain and body and severe suppression of proliferation. The underlying mechanisms, however, remain to be determined. CRT can inhibit growth through its action on the hypothalamus and/pituitary. Indeed, hypopituitarism is frequently observed in both adults [87-91] and children [84, 88, 92-94] treated with cranial radiation even for nonpituitary cancers. Animal studies also show that cranial radiation disrupts endocrine function in a dose-dependent manner [78, 95]. We are currently investigating to what extent our findings can be explained by the interaction of radiation and the endocrine system.

\section{Conclusions}

To our knowledge, this is the first report of the acute and long-term effects of CRT delivered during childhood/adolescence in an animal model which measures proliferation, microstructural metabolites (using translational imaging tools), and phenotype changes. Importantly, the observed early radiation-induced alterations were present $3.5 \mathrm{mo}$ after exposure coinciding with the early delayed phase of radiation injury. Ongoing investigations in our lab are aimed at evaluating the effects of fractionated CRT on cognition and the inhibition of the radiation-induced inflammatory response as a possible mechanism for mitigating the long-term sequelae.

\section{Competing Interests}

The authors declare that they have no competing interests regarding the publication of this paper.

\section{Authors' Contributions}

Shaefali P. Rodgers and Janice A. Zawaski are co-first authors with equal contributions.

\section{Acknowledgments}

This work was supported in part by a grant from the James S. McDonnell Foundation no. 1220020250 and the Cancer Prevention Institute of Texas RP130573CPRIT. The authors would like to thank Texas Children's Hospital for the use of the Small Animal Imaging Facility.

\section{References}

[1] J. A. Zawaski, M. W. Gaber, O. M. Sabek, C. M. Wilson, C. D. Duntsch, and T. E. Merchant, "Effects of irradiation on brain vasculature using an in situ tumor model," International Journal of Radiation Oncology, Biology, Physics, vol. 82, no. 3, pp. 10751082, 2012.

[2] P. Kaatsch, "Epidemiology of childhood cancer," Cancer Treatment Reviews, vol. 36, no. 4, pp. 277-285, 2010.

[3] G. Suneja, P. D. Poorvu, C. Hill-Kayser, and R. A. Lustig, "Acute toxicity of proton beam radiation for pediatric central nervous system malignancies," Pediatric Blood and Cancer, vol. 60, no. 9, pp. 1431-1436, 2013.

[4] P. D. Cole and B. A. Kamen, "Delayed neurotoxicity associated with therapy for children with acute lymphoblastic leukemia," Mental Retardation and Developmental Disabilities Research Reviews, vol. 12, no. 3, pp. 174-183, 2006.

[5] K. J. Redmond, E. M. Mahone, and A. Horska, "Association between radiation dose to neuronal progenitor cell niches and temporal lobes and performance on neuropsychological testing in children: a prospective study," Neuro-Oncology, vol. 15, no. 11, article 1455, 2013.

[6] R. J. Wells, M. B. Foster, A. J. D'Ercole, and C. W. McMillan, "The impact of cranial irradiation on the growth of children with acute lymphocytic leukemia," The American Journal of Diseases of Children, vol. 137, no. 1, pp. 37-39, 1983.

[7] G. E. Nam, S. Kaul, Y. P. Wu et al., "A meta-analysis of body mass index of adolescent and adult survivors of pediatric acute 
lymphoblastic leukemia," Journal of Cancer Survivorship, vol. 9, no. 3, pp. 412-421, 2015.

[8] J. Ochs, R. Mulhern, D. Fairclough et al., "Comparison of neuropsychologic functioning and clinical indicators of neurotoxicity in long-term survivors of childhood leukemia given cranial radiation or parenteral methotrexate: a prospective study," Journal of Clinical Oncology, vol. 9, no. 1, pp. 145-151, 1991.

[9] J. J. Ochs, "Neurotoxicity due to central nervous system therapy for childhood leukemia," Journal of Pediatric Hematology/Oncology, vol. 11, no. 1, pp. 93-105, 1989.

[10] M. Fouladi, M. Chintagumpala, F. H. Laningham et al., "White matter lesions detected by magnetic resonance imaging after radiotherapy and high-dose chemotherapy in children with medulloblastoma or primitive neuroectodermal tumor," Journal of Clinical Oncology, vol. 22, no. 22, pp. 4551-4560, 2004.

[11] R. K. Mulhern, S. L. Palmer, W. E. Reddick et al., "Risks of young age for selected neurocognitive deficits in medulloblastoma are associated with white matter loss," Journal of Clinical Oncology, vol. 19, no. 2, pp. 472-479, 2001.

[12] A. Kingma, E. L. Mooyaart, W. A. Kamps, P. Nieuwenhuizen, and J. T. Wilmink, "Magnetic resonance imaging of the brain and neuropsychological evaluation in children treated for acute lymphoblastic leukemia at a young age," American Journal of Pediatric Hematology/Oncology, vol. 15, no. 2, pp. 231-238, 1993.

[13] C. Hua, T. E. Merchant, A. Gajjar et al., "Brain tumor therapyinduced changes in normal-appearing brainstem measured with longitudinal diffusion tensor imaging," International Journal of Radiation Oncology Biology Physics, vol. 82, no. 5, pp. 2047-2054, 2012.

[14] S. L. Palmer, W. E. Reddick, J. O. Glass et al., "Regional white matter anisotropy and reading ability in patients treated for pediatric embryonal tumors," Brain Imaging and Behavior, vol. 4, no. 2, pp. 132-140, 2010.

[15] I. Schuitema, S. Deprez, W. Van Hecke et al., "Accelerated aging, decreased white matter integrity, and associated neuropsychological dysfunction 25 years after pediatric lymphoid malignancies," Journal of Clinical Oncology, vol. 31, no. 27, pp. 3378-3388, 2013.

[16] S. L. Palmer, W. E. Reddick, J. O. Glass, A. Gajjar, O. Goloubeva, and R. K. Mulhern, "Decline in corpus callosum volume among pediatric patients with medulloblastoma: longitudinal MR imaging study," American Journal of Neuroradiology, vol. 23, no. 7, pp. 1088-1094, 2002.

[17] W. E. Reddick, J. O. Glass, S. L. Palmer et al., "Atypical white matter volume development in children following craniospinal irradiation," Neuro-Oncology, vol. 7, no. 1, pp. 12-19, 2005.

[18] R. K. Mulhern, H. A. White, J. O. Glass et al., "Attentional functioning and white matter integrity among survivors of malignant brain tumors of childhood," Journal of the International Neuropsychological Society, vol. 10, no. 2, pp. 180-189, 2004.

[19] W. E. Reddick, R. K. Mulhern, T. David Elkin, J. O. Glass, T. E. Merchant, and J. W. Langston, "A hybrid neural network analysis of subtle brain volume differences in children surviving brain tumors," Magnetic Resonance Imaging, vol. 16, no. 4, pp. 413-421, 1998.

[20] W. E. Reddickaij, J. M. Russell, J. O. Glass et al., "Subtle white matter volume differences in children treated for medulloblastoma with conventional or reduced dose craniospinal irradiation," Magnetic Resonance Imaging, vol. 18, no. 7, pp. 787-793, 2000.

[21] M. N. Edelmann, K. R. Krull, W. Liu et al., "Diffusion tensor imaging and neurocognition in survivors of childhood acute lymphoblastic leukaemia," Brain, vol. 137, no. 11, pp. 2973-2983, 2014.

[22] T. Atwood, M. E. Robbins, and J.-M. Zhu, "Quantitative in vivo proton MR spectroscopic evaluation of the irradiated rat brain," Journal of Magnetic Resonance Imaging, vol. 26, no. 6, pp. 15901595, 2007.

[23] K. C. Chan, P.-I. Khong, M. M. Cheung, S. Wang, K.-X. Cai, and E. X. Wu, "MRI of late microstructural and metabolic alterations in radiation-induced brain injuries," Journal of Magnetic Resonance Imaging, vol. 29, no. 5, pp. 1013-1020, 2009.

[24] M. Gupta, P. Rana, R. Trivedi et al., "Comparative evaluation of brain neurometabolites and DTI indices following whole body and cranial irradiation: a magnetic resonance imaging and spectroscopy study," NMR in Biomedicine, vol. 26, no. 12, pp. 1733-1741, 2013.

[25] A. M. Peiffer, R. M. Creer, C. Linville et al., "Radiation-induced cognitive impairment and altered diffusion tensor imaging in a juvenile rat model of cranial radiotherapy," International Journal of Radiation Biology, vol. 90, no. 9, pp. 799-806, 2014.

[26] S. Wang, E. X. Wu, D. Qiu, L. H. T. Leung, H.-F. Lau, and P.L. Khong, "Longitudinal diffusion tensor magnetic resonance imaging study of radiation-induced white matter damage in a rat model," Cancer Research, vol. 69, no. 3, pp. 1190-1198, 2009.

[27] L. Adan, C. Trivin, C. Sainte-Rose, J. M. Zucker, O. Hartmann, and R. Brauner, "GH deficiency caused by cranial irradiation during childhood: factors and markers in young adults," The Journal of Clinical Endocrinology \& Metabolism, vol. 86, no. 11, pp. 5245-5251, 2001.

[28] A. G. Goddard, S. J. Harris, P. N. Plowman, M. O. Savage, and J. E. Kingston, "Growth hormone deficiency following radiotherapy for orbital and parameningeal sarcomas," Pediatric Hematology and Oncology, vol. 16, no. 1, pp. 23-33, 1999.

[29] L. R. Meacham, P. W. Mason, and K. M. Sullivan, "Auxologic and biochemical characterization of the three phases of growth failure in pediatric patients with brain tumors," Journal of Pediatric Endocrinology and Metabolism, vol. 17, no. 5, pp. 711717, 2004.

[30] N. Borrmann, S. Friedrich, K. Schwabe et al., "Systemic treatment with 4-211At-phenylalanine enhances survival of rats with intracranial glioblastoma," Nuklearmedizin, vol. 52, no. 6, pp. 212-221, 2013.

[31] L. Iughetti, P. Bruzzi, B. Predieri, and P. Paolucci, "Obesity in patients with acute lymphoblastic leukemia in childhood," Italian Journal of Pediatrics, vol. 38, article 4, 2012.

[32] K. R. Wolfe, G. R. Hunter, A. Madan-Swain, A. T. Reddy, J. Baños, and R. K. Kana, "Cardiorespiratory fitness in survivors of pediatric posterior fossa tumor," Journal of Pediatric Hematology/Oncology, vol. 34, no. 6, pp. e222-e227, 2012.

[33] L. K. Zeltzer, C. Recklitis, D. Buchbinder et al., "Psychological status in childhood cancer survivors: a report from the childhood cancer survivor study," Journal of Clinical Oncology, vol. 27, no. 14, pp. 2396-2404, 2009.

[34] N. J. Ullrich and L. Embry, "Neurocognitive dysfunction in survivors of childhood brain tumors," Seminars in Pediatric Neurology, vol. 19, no. 1, pp. 35-42, 2012.

[35] M. W. Gaber, O. M. Sabek, K. Fukatsu, H. G. Wilcox, M. F. Kiani, and T. E. Merchant, "Differences in ICAM-1 and TNF$\alpha$ expression between large single fraction and fractionated irradiation in mouse brain," International Journal of Radiation Biology, vol. 79, no. 5, pp. 359-366, 2003.

[36] J. Raber, R. Rola, A. LeFevour et al., "Radiation-induced cognitive impairments are associated with changes in indicators of 
hippocampal neurogenesis," Radiation Research, vol. 162, no. 1, pp. 39-47, 2004.

[37] R. Rola, J. Raber, A. Rizk et al., "Radiation-induced impairment of hippocampal neurogenesis is associated with cognitive deficits in young mice," Experimental Neurology, vol. 188, no. 2, pp. 316-330, 2004.

[38] M. Andres-Mach, R. Rola, and J. R. Fike, "Radiation effects on neural precursor cells in the dentate gyrus," Cell and Tissue Research, vol. 331, no. 1, pp. 251-262, 2008.

[39] B. Milner, “The medial temporal-lobe amnesic syndrome," Psychiatric Clinics of North America, vol. 28, no. 3, pp. 599-611, 2005.

[40] A. R. Preston and H. Eichenbaum, "Interplay of hippocampus and prefrontal cortex in memory," Current Biology, vol. 23, no. 17, pp. R764-R773, 2013.

[41] M. Monje and J. Dietrich, "Cognitive side effects of cancer therapy demonstrate a functional role for adult neurogenesis," Behavioural Brain Research, vol. 227, no. 2, pp. 376-379, 2012.

[42] M. L. Monje, H. Vogel, M. Masek, K. L. Ligon, P. G. Fisher, and T. D. Palmer, "Impaired human hippocampal neurogenesis after treatment for central nervous system malignancies," Annals of Neurology, vol. 62, no. 5, pp. 515-520, 2007.

[43] M. Monje, "Cranial radiation therapy and damage to hippocampal neurogenesis," Developmental Disabilities Research Reviews, vol. 14, no. 3, pp. 238-242, 2008.

[44] S. P. Rodgers, M. Trevino, J. A. Zawaski, M. W. Gaber, and J. L. Leasure, "Neurogenesis, exercise, and cognitive late effects of pediatric radiotherapy," Neural Plasticity, vol. 2013, Article ID 698528, 12 pages, 2013.

[45] M. E. Maynard and J. L. Leasure, "Exercise enhances hippocampal recovery following binge ethanol exposure," PLoS ONE, vol. 8, no. 9, Article ID e76644, 2013.

[46] W. Peißner, M. Kocher, H. Treuer, and F. Gillardon, "Ionizing radiation-induced apoptosis of proliferating stem cells in the dentate gyrus of the adult rat hippocampus," Molecular Brain Research, vol. 71, no. 1, pp. 61-68, 1999.

[47] D. M. Curlik II, G. DiFeo, and T. J. Shors, "Preparing for adulthood: thousands upon thousands of new cells are born in the hippocampus during puberty, and most survive with effortful learning," Frontiers in Neuroscience, vol. 8, article 70, 2014.

[48] J. R. Epp, J. M. Barker, and L. A. M. Galea, "Running wild: neurogenesis in the hippocampus across the lifespan in wild and laboratory-bred Norway rats," Hippocampus, vol. 19, no. 10, pp. 1040-1049, 2009.

[49] K. Roughton, M. Boström, M. Kalm, and K. Blomgren, "Irradiation to the young mouse brain impaired white matter growth more in females than in males," Cell Death and Disease, vol. 4, no. 10, article e897, 2013.

[50] G. Stratmann, J. W. Sall, L. D. V. May et al., "Isoflurane differentially affects neurogenesis and long-term neurocognitive function in 60-day-old and 7-day-old rats," Anesthesiology, vol. 110, no. 4, pp. 834-848, 2009.

[51] C. Zhu, J. Gao, N. Karlsson et al., "Isoflurane anesthesia induced persistent, progressive memory impairment, caused a loss of neural stem cells, and reduced neurogenesis in young, but not adult, rodents," Journal of Cerebral Blood Flow and Metabolism, vol. 30, no. 5, pp. 1017-1030, 2010.

[52] S. Mizumatsu, M. L. Monje, D. R. Morhardt, R. Rola, T. D. Palmer, and J. R. Fike, "Extreme sensitivity of adult neurogenesis to low doses of X-irradiation," Cancer Research, vol. 63, no. 14, pp. 4021-4027, 2003.
[53] P. Achanta, M. Fuss, and J. L. Martinez Jr., "Ionizing radiation impairs the formation of trace fear memories and reduces hippocampal neurogenesis," Behavioral Neuroscience, vol. 123, no. 5, pp. 1036-1045, 2009.

[54] K. A. Jenrow, S. L. Brown, K. Lapanowski, H. Naei, A. Kolozsvary, and J. H. Kim, "Selective inhibition of microgliamediated neuroinflammation mitigates radiation-induced cognitive impairment," Radiation Research, vol. 179, no. 5, pp. 549556, 2013.

[55] M. Boström, N. Hellström Erkenstam, D. Kaluza, L. Jakobsson, M. Kalm, and K. Blomgren, "The hippocampal neurovascular niche during normal development and after irradiation to the juvenile mouse brain," International Journal of Radiation Biology, vol. 90, no. 9, pp. 778-789, 2014.

[56] A. Barlind, N. Karlsson, T. Björk-Eriksson, J. Isgaard, and K. Blomgren, "Decreased cytogenesis in the granule cell layer of the hippocampus and impaired place learning after irradiation of the young mouse brain evaluated using the IntelliCage platform," Experimental Brain Research, vol. 201, no. 4, pp. 781787, 2010.

[57] N. A. K. Hellström, T. Björk-Eriksson, K. Blomgren, and H. G. Kuhn, "Differential recovery of neural stem cells in the subventricular zone and dentate gyrus after ionizing radiation," STEM CELLS, vol. 27, no. 3, pp. 634-641, 2009.

[58] A. A. Nageswara Rao, H. Ye, P. A. Decker, C. L. Howe, and C. Wetmore, "Therapeutic doses of cranial irradiation induce hippocampus-dependent cognitive deficits in young mice," Journal of Neuro-Oncology, vol. 105, no. 2, pp. 191-198, 2011.

[59] I. Conlon and M. Raff, "Size control in animal development," Cell, vol. 96, no. 2, pp. 235-244, 1999.

[60] J. Liu, X. Ji, Z. Li et al., "Nestin overexpression promotes the embryonic development of heart and brain through the regulation of cell proliferation," Brain Research, vol. 1610, pp. 1-11, 2015.

[61] T. J. Shors, G. Miesegaes, A. Beylin, M. Zhao, T. Rydel, and E. Gould, "Neurogenesis in the adult is involved in the formation of trace memories," Nature, vol. 410, no. 6826, pp. 372-376, 2001.

[62] G. E. Wood, A. V. Beylin, and T. J. Shors, "The contribution of adrenal and reproductive hormones to the opposing effects of stress on trace conditioning in males versus females," Behavioral Neuroscience, vol. 115, no. 1, pp. 175-187, 2001.

[63] A. L. Alexander, J. E. Lee, M. Lazar, and A. S. Field, "Diffusion tensor imaging of the brain," Neurotherapeutics, vol. 4, no. 3, pp. 316-329, 2007.

[64] Y. Assaf and O. Pasternak, "Diffusion tensor imaging (DTI)based white matter mapping in brain research: a review," Journal of Molecular Neuroscience, vol. 34, no. 1, pp. 51-61, 2008.

[65] S. Wang, D. Qiu, K.-F. So et al., "Radiation induced brain injury: assessment of white matter tracts in a pre-clinical animal model using diffusion tensor MR imaging," Journal of Neuro-Oncology, vol. 112, no. 1, pp. 9-15, 2013.

[66] R. Trivedi, A. R. Khan, P. Rana et al., "Radiation-induced early changes in the brain and behavior: serial diffusion tensor imaging and behavioral evaluation after graded doses of radiation," Journal of Neuroscience Research, vol. 90, no. 10, pp. 2009-2019, 2012.

[67] W. Calvo, J. W. Hopewell, H. S. Reinhold, and T. K. Yeung, "Time- and dose-related changes in the white matter of the rat brain after single doses of X rays," British Journal of Radiology, vol. 61, no. 731, pp. 1043-1052, 1988.

[68] K. Akiyama, R. Tanaka, M. Sato, and N. Takeda, "Cognitive dysfunction and histological findings in adult rats one year after 
whole brain irradiation," Neurologia Medico-Chirurgica, vol. 41, no. 12, pp. 590-598, 2001.

[69] H. Hodges, N. Katzung, P. Sowinski et al., "Late behavioural and neuropathological effects of local brain irradiation in the rat," Behavioural Brain Research, vol. 91, no. 1-2, pp. 99-114, 1998.

[70] A. E. de Guzman, L. M. Gazdzinski, R. J. Alsop et al., "Treatment age, dose and sex determine neuroanatomical outcome in irradiated juvenile mice," Radiation Research, vol. 183, no. 5, pp. 541-549, 2015.

[71] C. E. Mountford, P. Stanwell, A. Lin, S. Ramadan, and B. Ross, "Neurospectroscopy: the past, present and future," Chemical Reviews, vol. 110, no. 5, pp. 3060-3086, 2010.

[72] A. Panigrahy, M. D. Nelson Jr., and S. Blüml, "Magnetic resonance spectroscopy in pediatric neuroradiology: clinical and research applications," Pediatric Radiology, vol. 40, no. 1, pp. 330, 2010.

[73] T. Atwood, V. S. Payne, W. Zhao et al., "Quantitative magnetic resonance spectroscopy reveals a potential relationship between radiation-induced changes in rat brain metabolites and cognitive impairment," Radiation Research, vol. 168, no. 5, pp. 574581, 2007.

[74] H. Yuan, M. W. Gaber, K. Boyd, C. M. Wilson, M. F. Kiani, and T. E. Merchant, "Effects of fractionated radiation on the brain vasculature in a murine model: blood-brain barrier permeability, astrocyte proliferation, and ultrastructural changes," International Journal of Radiation Oncology, Biology, Physics, vol. 66, no. 3, pp. 860-866, 2006.

[75] C. M. Wilson, M. W. Gaber, O. M. Sabek, J. A. Zawaski, and T. E. Merchant, "Radiation-induced astrogliosis and blood-brain barrier damage can be abrogated using anti-TNF treatment," International Journal of Radiation Oncology Biology Physics, vol. 74, no. 3, pp. 934-941, 2009.

[76] Y. Tian, Z. Shi, S. Yang, Y. Chen, and S. Bao, "Changes in myelin basic protein and demyelination in the rat brain within 3 months of single 2-, 10-, or 30-Gy whole-brain radiation treatments," Journal of Neurosurgery, vol. 109, no. 5, pp. 881-888, 2008.

[77] C.-S. Chiang, W. H. McBride, and H. Rodney Withers, "Myelinassociated changes in mouse brain following irradiation," Radiotherapy and Oncology, vol. 27, no. 3, pp. 229-236, 1993.

[78] I. C. A. F. Robinson, K. M. Fairhall, J. H. Hendry, and S. M. Shalet, "Differential radiosensitivity of hypothalamo-pituitary function in the young adult rat," Journal of Endocrinology, vol. 169, no. 3, pp. 519-526, 2001.

[79] X. Z. Sun, M. Inouye, S. Hayasaka, and H. Yamamura, "Body and brain development following exposure to 60Co gammairradiation during pregnancy in mice," Environmental Medicine, vol. 38, no. 2, pp. 111-114, 1994.

[80] A. Schunior, A. E. Zengel, P. J. Mullenix, N. J. Tarbella, A. Howes, and M. S. Tassinari, "An animal model to study toxicity of central nervous system therapy for childhood acute lymphoblastic leukemia: effects on growth and craniofacial proportion," Cancer Research, vol. 50, no. 20, pp. 6455-6460, 1990.

[81] A. Schunior, P. J. Mullenix, A. E. Zengel, H. Landy, A. Howes, and N. J. Tarbell, "Radiation effects on growth are altered in rats by prednisolone and methotrexate," Pediatric Research, vol. 35, no. 4, part 1, pp. 416-423, 1994.

[82] H. D. Mosier Jr., R. A. Jansons, K. F. Swingle, C. A. Sondhaus, L. C. Dearden, and L. C. Halsall, "Growth hormone secretion in the stunted head-irradiated rat," Pediatric Research, vol. 19, no. 6, pp. 543-548, 1985.
[83] T. Amano, T. Inamura, C.-M. Wu et al., "Effects of single low dose irradiation on subventricular zone cells in juvenile rat brain," Neurological Research, vol. 24, no. 8, pp. 809-816, 2002.

[84] D. Beckers, M. Thomas, J. Jamart et al., "Adult final height after GH therapy for irradiation-induced GH deficiency in childhood survivors of brain tumors: the Belgian experience," European Journal of Endocrinology, vol. 162, no. 3, pp. 483-490, 2010.

[85] E. A. Schriock, M. J. Schell, M. Carter, O. Hustu, and J. J. Ochs, "Abnormal growth patterns and adult short stature in 115 longterm survivors of childhood leukemia," Journal of Clinical Oncology, vol. 9, no. 3, pp. 400-405, 1991.

[86] V. Sulmont, R. Brauner, M. Fontoura, and R. Rappaport, "Response to growth hormone treatment and final height after cranial or craniospinal irradiation," Acta Paediatrica Scandinavica, vol. 79, no. 5, pp. 542-549, 1990.

[87] K. H. Darzy, "Radiation-induced hypopituitarism," Current Opinion in Endocrinology, Diabetes and Obesity, vol. 20, no. 4, pp. 342-353, 2013.

[88] T. Sathyapalan and S. Dixit, "Radiotherapy-induced hypopituitarism: a review," Expert Review of Anticancer Therapy, vol. 12, no. 5, pp. 669-683, 2012.

[89] N. M. Appelman-Dijkstra, F. Malgo, K. J. Neelis, I. Coremans, N. R. Biermasz, and A. M. Pereira, "Pituitary dysfunction in adult patients after cranial irradiation for head and nasopharyngeal tumours," Radiotherapy and Oncology, vol. 113, no. 1, pp. 102107, 2014.

[90] N. M. Appelman-Dijkstra, N. E. Kokshoorn, O. M. Dekkers et al., "Pituitary dysfunction in adult patients after cranial radiotherapy: systematic review and meta-analysis," The Journal of Clinical Endocrinology \& Metabolism, vol. 96, no. 8, pp. 23302340, 2011.

[91] A. Agha, M. Sherlock, S. Brennan et al., "Hypothalamicpituitary dysfunction after irradiation of nonpituitary brain tumors in adults," Journal of Clinical Endocrinology and Metabolism, vol. 90, no. 12, pp. 6355-6360, 2005.

[92] W. Chemaitilly, Z. Li, S. Huang et al., "Anterior hypopituitarism in adult survivors of childhood cancers treated with cranial radiotherapy: a report from the St Jude Lifetime Cohort study," Journal of Clinical Oncology, vol. 33, no. 5, pp. 492-500, 2015.

[93] V. Viswanathan, K. R. Pradhan, and E. A. Eugster, "Pituitary hormone dysfunction after proton beam radiation therapy in children with brain tumors," Endocrine Practice, vol. 17, no. 6, pp. 891-896, 2011.

[94] G. E. Richards, W. M. Wara, M. M. Grumbach, S. L. Kaplan, G. E. Sheline, and F. A. Conte, "Delayed onset of hypopituitarism: sequelae of therapeutic irradiation of central nervous system, eye, and middle ear tumors," The Journal of Pediatrics, vol. 89, no. 4, pp. 553-559, 1976.

[95] C. Roth, H. Schmidberger, O. Schaper et al., "Cranial irradiation of female rats causes dose-dependent and age-dependent activation or inhibition of pubertal development," Pediatric Research, vol. 47, no. 5, pp. 586-591, 2000. 

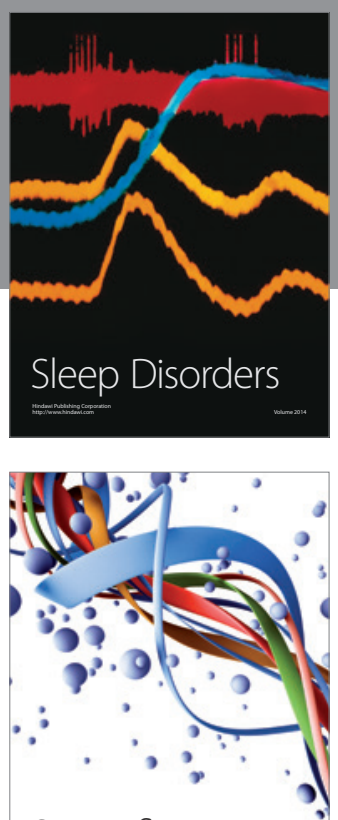

Scientifica
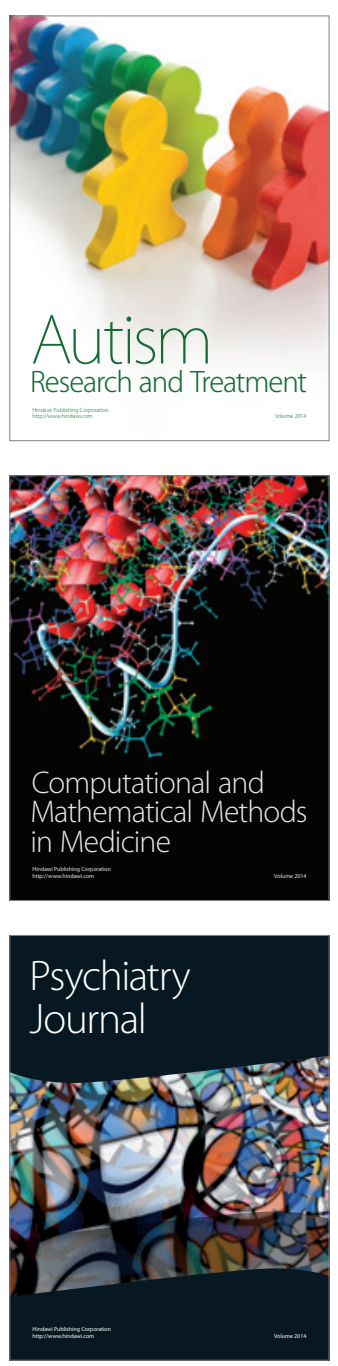
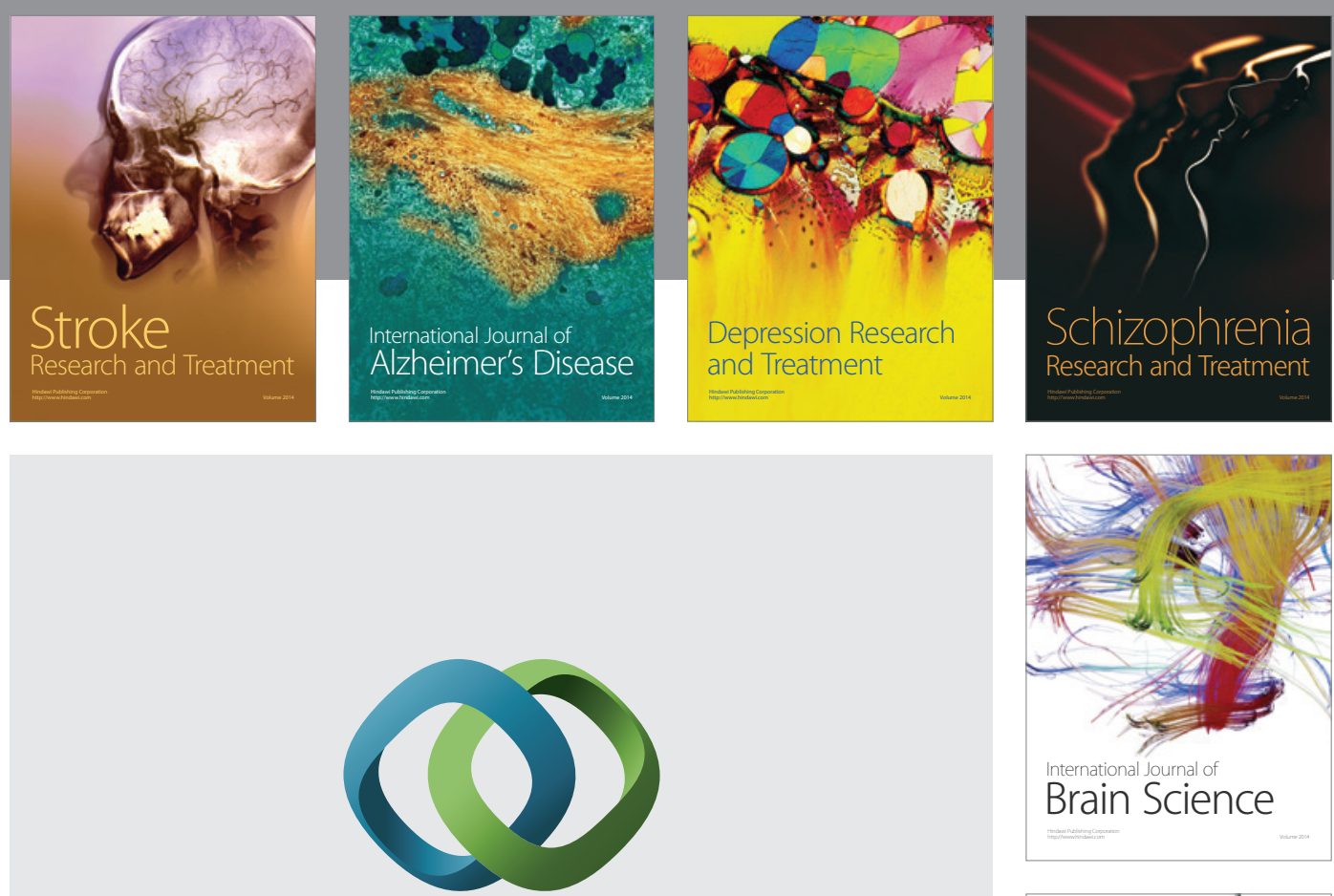

\section{Hindawi}

Submit your manuscripts at

http://www.hindawi.com
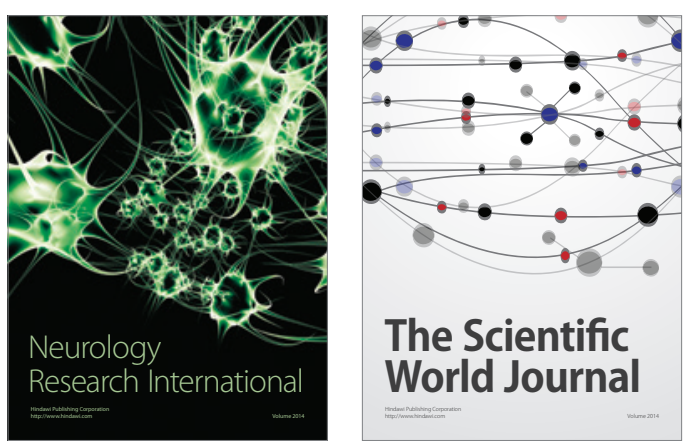

The Scientific World Journal

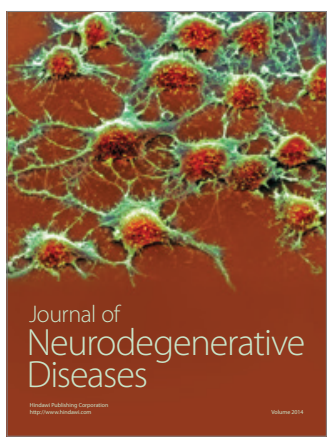

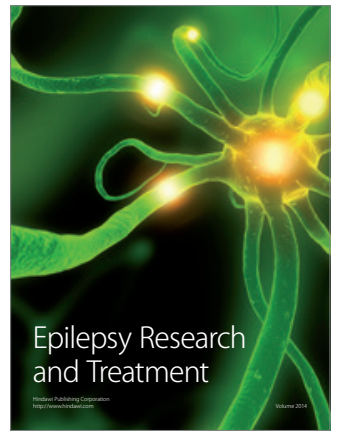

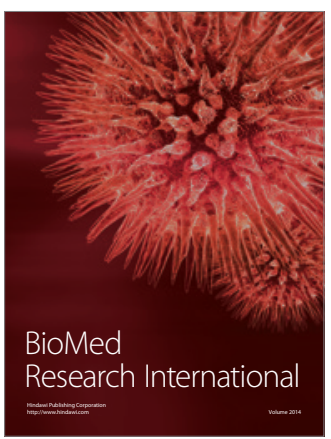

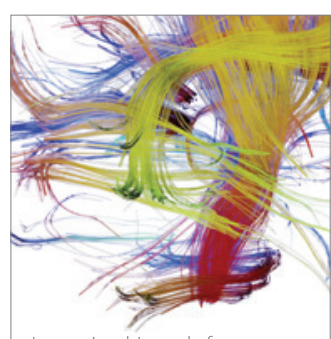

Brain Science

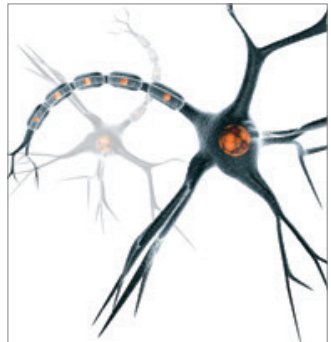

Neural Plasticity
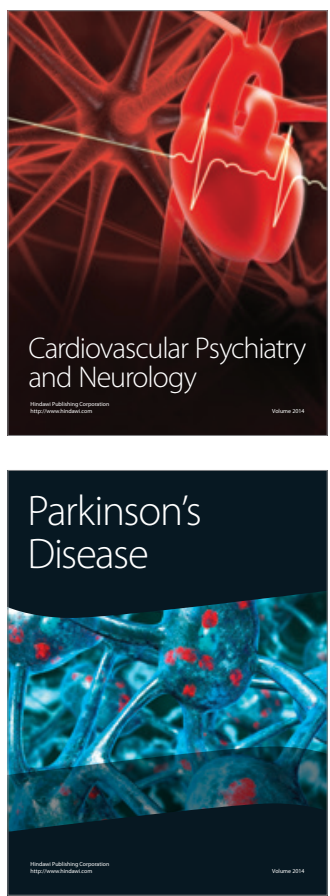